\title{
Left Ventricular Longitudinal Myocardial Contraction Is Associated with Cognitive Status: A Cross-Sectional Study in Community-Dwelling Populations
}

liu yongtai ${ }^{1}$, lai jinzhi ${ }^{2}$, zhai feifei ${ }^{2}$, han fei ${ }^{2}$, zhou lixin ${ }^{2}$, ni jun ${ }^{2}$, yao ming $^{2}$, Zhuang $\operatorname{Tian}^{3}$, zhu yanlin ${ }^{2}$, chen wei ${ }^{2}$, bai hua ${ }^{2}$, wang hui ${ }^{2}$, zhang dingding ${ }^{2}$, cui liying ${ }^{2}$, jin zhengyu ${ }^{2}$, zhang shuyang ${ }^{2}$, and zhu yicheng ${ }^{2}$

${ }^{1}$ Affiliation not available

${ }^{2}$ Peking Union Medical College Hospital

${ }^{3}$ Peking Union Medical College Hospital, Chinese Academy of Medical Sciences and Peking Union Medical College

September 21, 2020

\begin{abstract}
Aims: The interaction between the heart and brain is highly complex. Left ventricular (LV) longitudinal contraction is the most sensitive to the presence of myocardial disease. Whether subclinical change in LV longitudinal contraction assessed by echocardiography is associated with cognitive impairment in the general population has not been investigated. Methods and Results: All residents aged [?] 35 years, living in five villages of Shunyi, a suburb district of Beijing, were invited to participate in the study from June 2013 to April 2016. This was an exploratory cross-sectional analysis of the baseline data of 894 participants enrolled in the Shunyi Study. Cognitive status was evaluated using the Montreal Cognitive Assessment (MoCA). LV longitudinal contraction was assessed by the peak systolic velocity measured at the mitral annulus (Sm) obtained by Tissue Doppler imaging echocardiography. Cognitive impairment (MoCA score <26) was found in 771 (86.2\%) participants. LV longitudinal myocardial contraction $(\mathrm{Sm})$ and diastolic function (Em, E/A ratio, and E/e?) were associated with cognitive impairment (all $\mathrm{P}<0.01$ ). The association between $\mathrm{Sm}$ and cognitive impairment remained significant after adjusting for age, sex, education level, physical activity, vascular risk factors, and cerebral small-vessel disease (OR, 0.84; 95\% CI, 0.73-0.97, $\mathrm{P}=0.02)$. Receiver operating characteristic curve analysis for $\mathrm{Sm}$ in identifying normal cognitive status showed the area under the curve of 0.59 (95\% CI, 0.54-0.65; P<0.01). Conclusions: Our findings suggest that LV longitudinal myocardial contraction in the general population is associated with cognitive status in Chinese community-dwelling populations.
\end{abstract}

\section{Hosted file}

manuscript-revised-with tables.doc available at https://authorea.com/users/360557/

articles/482192-left-ventricular-longitudinal-myocardial-contraction-is-associated-withcognitive-status-a-cross-sectional-study-in-community-dwelling-populations 
1,586 individuals

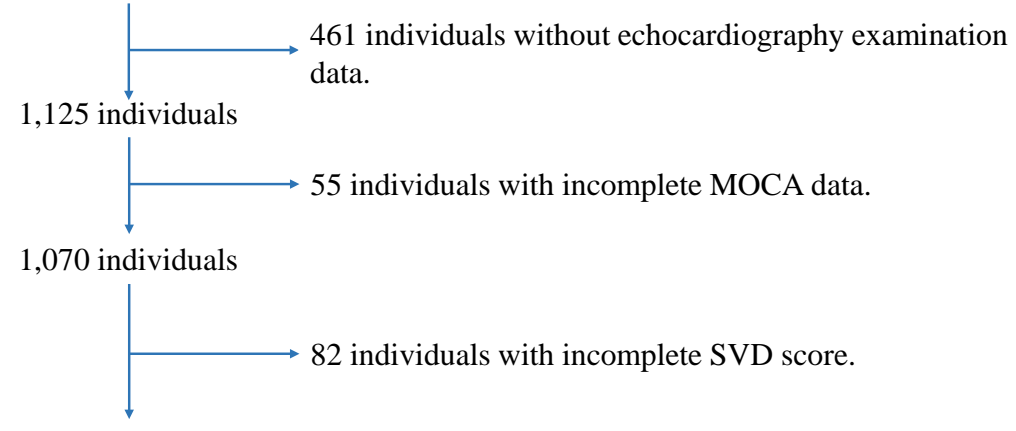

998 individuals

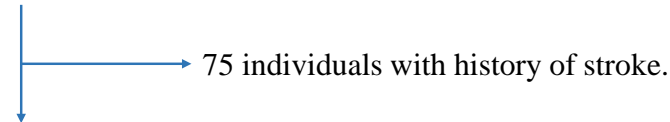

913 individuals

$\longrightarrow 19$ individuals with poor echocardiography image quality.

894 individuals were included in the analysis

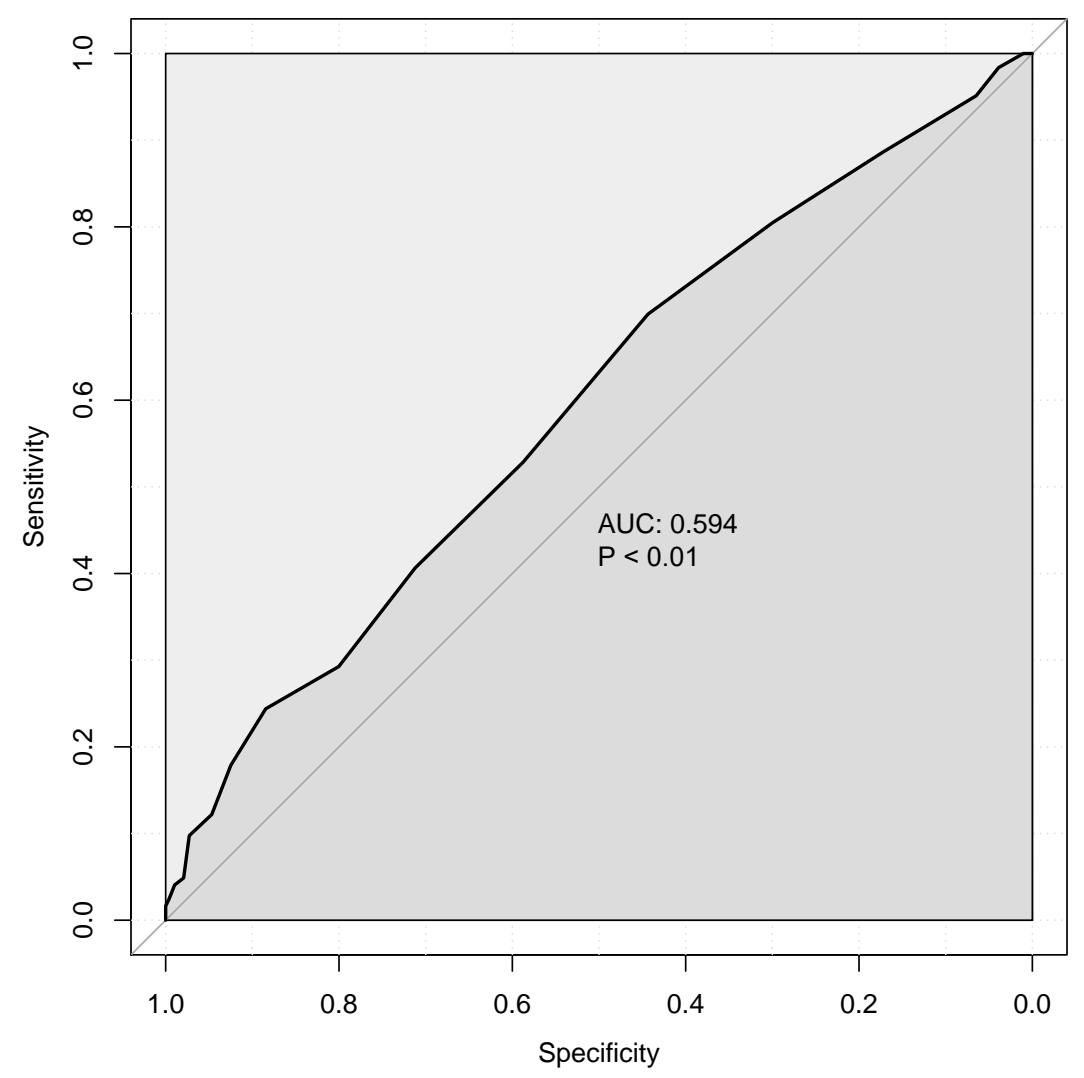

